\title{
P 101 DO HOSPITAL NURSES ADMINISTER NASAL FENTANYL SPRAY CORRECTLY? - A SERVICE EVALUATION
}

Samuel Fingas, ${ }^{1}$ Anne-Marie Bourke, ${ }^{1}$ Vanessa Spawton, ${ }^{1}$ Sian Richardson, ${ }^{1}$ Elizabeth Miller, 2,1 Sam H Ahmedzai ${ }^{3,1}$. 'Sheffield Teaching Hospitals, Sheffield, United Kingdom; ${ }^{2}$ St Luke's Hospice, Sheffield, United Kingdom; ${ }^{3}$ University of Sheffield, Sheffield, United Kingdom

\subsection{6/bmjspcare-2014-000654.142}

Background PecFent ${ }^{\circledR}$ is a powerful fast-acting fentanyl nasal spray which is used for breakthrough cancer pain. When it is not administered correctly or titrated quickly enough, we suspect aspects of patient care may suffer, with sub-optimal pain control, loss of confidence in the medication, and unnecessary cost to the health service. Aims To assess hospital nurses' confidence and competence in administering PecFent ${ }^{\circledR}$. To identify specific needs for further training.

Methods We used a convenience sample of 5 nurses (who had previously administered PecFent ${ }^{\circledR}$ ) per ward or hospice unit in Sheffield where PecFent ${ }^{\circledR}$ is commonly prescribed. A guided questionnaire-based interview, involving a standardised scenario using placebo PecFent ${ }^{\circledR}$, was piloted on 4 specialist nurses. The scenario required the nurse to titrate the dose from 100 to $200 \mathrm{mcg}$ and administer it to an actor, followed by questions on confidence, previous experience, training, and understanding of PecFent ${ }^{\circledR}$.

Results A total of 35 nurses were interviewed from 7 wards/units. $29 \%(10 / 35)$ chose the correct dose of $200 \mathrm{mcg}$. Commonest reasons for reluctance to titrate the dose up were: "long interval since previous dose" $(\mathrm{n}=16)$; and "only had $100 \mathrm{mcg}$ doses previously" ( $n=8)$. Only $40 \%$ enquired about effectiveness of the previous dose. $34 \%$ gave a spray effectively (primed device, aiming at nasal septum, no deep inhalation, one nostril/spray). $6 \%$ gave the correct dose effectively. $69 \%$ of nurses had given PecFent ${ }^{\circledR}>10$ times previously. $54 \%$ rated their confidence as $\leq 7 / 10$. Only one nurse had received formal training; $80 \%$ of nurses had learnt through observing a colleague. $77 \%$ wanted further training.

Conclusion We have demonstrated lack of confidence and competence in administering and titrating PecFent ${ }^{\circledR}$ effectively. We are making a training video to cover priming, direction of spay, instructions for breathing, and dispelling the myths about titration. 